\begin{tabular}{|c|l|}
\hline Title & Droplet coalescence process under electric fields in an immiscible polymer blend \\
\hline Author(s) & Aida, Kohei; Na, Y ang Ho; Nagaya, Tomoyuki; Orihara, Hiroshi \\
\hline Citation & $\begin{array}{l}\text { Physical Review E, 82(3), 031805 } \\
\text { https://doi.org/_0.1103/PhysRevE.82.031805 }\end{array}$ \\
\hline Issue Date & 2010-09 \\
\hline Doc URL & http://hdl.handle.net/2115/44032 \\
\hline Rights & ○2010 The A merican Physical Society \\
\hline Type & article \\
\hline File Information & PRE82-3_031805.pdf \\
\hline
\end{tabular}

Instructions for use 


\title{
Droplet coalescence process under electric fields in an immiscible polymer blend
}

\author{
Kohei Aida, ${ }^{1}$ Yang Ho Na, ${ }^{1}$ Tomoyuki Nagaya, ${ }^{2}$ and Hiroshi Orihara ${ }^{1, *}$ \\ ${ }^{1}$ Division of Applied Physics, Faculty of Engineering, Hokkaido University, Sapporo, Hokkaido 060-8628, Japan \\ ${ }^{2}$ Department of Electrical and Electronic Engineering, Faculty of Engineering, Oita University, Oita 870-1192, Japan
}

(Received 22 June 2010; revised manuscript received 2 September 2010; published 28 September 2010)

\begin{abstract}
The droplet coalescence process was investigated in immiscible polymer blends when subjected to a step electric field. We present sequential three-dimensional images captured during the process with a confocal scanning laser microscope. Characteristic lengths parallel and perpendicular to the electric field were obtained from the spatial correlation functions of the images. It was found that the droplet growth rate increased with both the electric field and the volume fraction of droplets. A function describing the droplet growth rate was derived from theory using the "hierarchical model" and was found to be in good agreement with the experimental results.
\end{abstract}

DOI: 10.1103/PhysRevE.82.031805

PACS number(s): $83.80 . \mathrm{Tc}, 82.70 . \mathrm{Kj}, 83.10 . \mathrm{TV}$

\section{INTRODUCTION}

The electrorheological (ER) effect in immiscible polymer blends, where a large viscosity change is induced when an electric field is applied, has attracted much interest from both the fundamental and application sides of science. The ER effect is closely related to morphological changes [1-6], which may be caused by shear flow and Maxwell stresses arising from mismatch of electric properties such as permittivity and conductivity between the two phases. In general, the ER effect appears under shear flow, but it has been found that columns are also formed along the electric field without shear flow, and the elastic modulus of the columnar structure increases with the electric field [7]. In our previous paper [7], we demonstrated a new system combining a confocal laser scanning microscope (CLSM) and a rheometer that enabled simultaneous three-dimensional (3D) observations and rheological measurements. From this work, it was seen that the elongation and coalescence of droplets appeared to play important roles in the formation process of the columnar structure under electric fields; hence, we now turn our attention to these processes.

One proposed application for these materials is, for example, using the coalescence under electric fields to remove water droplets from oils. Several studies have observed experimentally the coalescence process of a pair of droplets subjected to an electric field in order to elucidate the coalescence mechanism [8-10]. However, to the extent of our knowledge, the coalescence process of multiple droplets has not been reported to date.

In colloidal systems, on the other hand, the formation process of the columnar structure (chain or bridge) under electric fields, where clusters consisting of particles grow larger with time via aggregation [11-14], has been well studied. Furthermore, this formation process is well understood in terms of the hierarchical model [15] to be described later. The droplet coalescence process may be similar to the aggregation process of the colloidal particles, which implies that this model may be applicable to the immiscible polymer

*orihara@eng.hokudai.ac.jp blends studied here. In the present paper, we first present sequential 3D images captured during the droplet coalescence process after applying a step electric field. Then we derive the time dependence of the average droplet size from spatial correlation functions from the images, and from the results obtained by changing the strength of the electric field applied, a scaling property is derived. Lastly, we show that the hierarchical model can successfully represent the time evolution of the droplet coalescence process as observed experimentally.

\section{EXPERIMENT}

We used an immiscible polymer blend of a liquidcrystalline polymer (LCP) [16] (Fig. 1) and polyisobutylene (PIB). All the experiments were performed at $25^{\circ} \mathrm{C}$, and LCP was in the isotropic phase at this temperature. However, it should be noted that mesogens of LCP play a role in enhancing the permittivity and conductivity. We used PIB because it has low permittivity and conductivity in comparison to LCP, and the refractive indices of the fluids are closely matched, which is necessary for the observation of the structures with a CSLM. The viscosity, relative dielectric constant, conductivity, and refractive index of LCP at $25^{\circ} \mathrm{C}$ are $65 \mathrm{~Pa} \mathrm{~s}, 15,4 \times 10^{-9} \Omega^{-1} \mathrm{~m}^{-1}$, and 1.486 , respectively; those of PIB are $7.8 \mathrm{~Pa} \mathrm{~s}, 2.6,5 \times 10^{-11} \Omega^{-1} \mathrm{~m}^{-1}$, and 1.485, respectively. For the CSLM observations a small amount of fluorescent dye, IANBD amide (Molecular

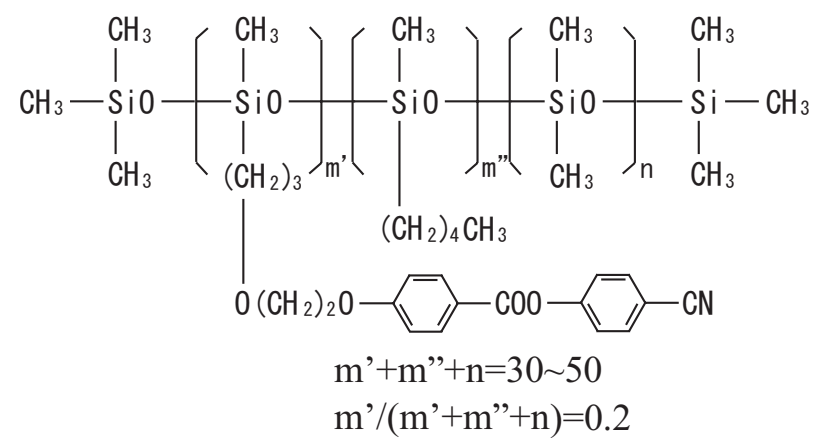

FIG. 1. Chemical structure of LCP. 


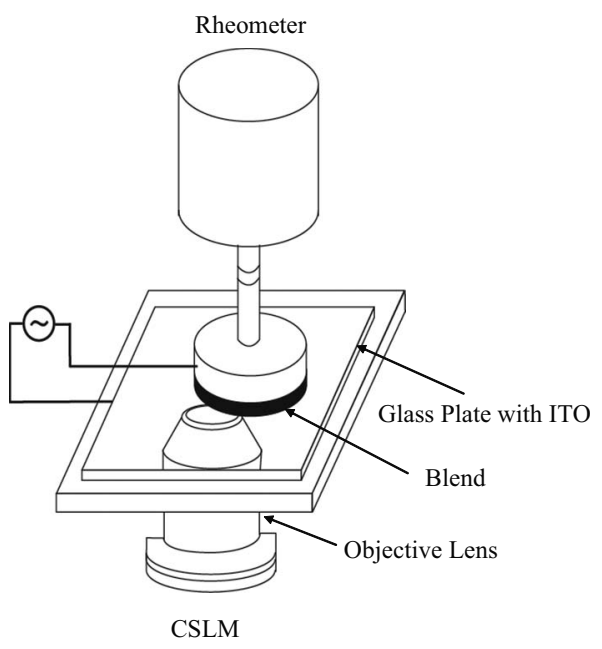

FIG. 2. Schematic of the system combining a CSLM and a rheometer.

Probes), was added to LCP before mixing with PIB. It was confirmed that the dye did not dissolve in PIB. The wavelength of excitation was $488 \mathrm{~nm}$, and a filter of $570 \mathrm{~nm}$ for the detection of light was used. Mixtures were made with volume ratios of $\mathrm{LCP}: \mathrm{PIB}=1: 6,1: 10$, and $1: 14$. In the absence of an electric field, LCP was dispersed in PIB as droplets in this blend.

A schematic of the system is shown in Fig. 2. The sample was sandwiched between a bottom glass plate with an ITOcoated electrode and the rotating metal plate of a rheometer (MCR301, Anton Paar). The diameter of the rotating plate was $35 \mathrm{~mm}$, and the gap between the plates was $200 \mu \mathrm{m}$. Observations were made through the bottom glass plate with a CSLM (IX71, Olympus and CSU22, Yokogawa). To avoid ionic migration we applied ac electric fields of $512 \mathrm{~Hz}$ via a synthesizer (Multifunction Synthesizer 1940, NF Electric Instruments) and a high voltage amplifier (model 609C-6, Trek). We constructed 3D images by using a piezoactuator (P-721.10, PI) attached to the objective lens for vertical displacement. We applied a triangular voltage of $0.3 \mathrm{~Hz}$ to the piezoactuator, so that the objective lens or the focal plane of the microscope oscillated vertically between 32 and $145 \mu \mathrm{m}$ above the bottom glass surface. The frame rate of the CSLM was 60 frames per second, so that 100 images were captured per half period, from which one 3D image was constructed. We successfully obtained clear 3D animations.

\section{RESULTS}

In the absence of an electric field LCP was dispersed in PIB as droplets in this polymer blend. Before each measurement a preshear was applied for $20 \mathrm{~min}$ at $200 \mathrm{~s}^{-1}$ to attain a steady state. Figures 3(a) and 3(b) show the sequential 3D images obtained at (a) 2 and (b) $4 \mathrm{kV}_{\mathrm{amp}} / \mathrm{mm}$, for $\phi=0.14$ (LCP:PIB =1:6), where the subscript in "V" indicates the amplitude of the ac voltages (see movies 1 and 2 [17]). At $t=0$ before applying the electric field without shear, the droplet radius was $\sim 2 \mu \mathrm{m}$. Immediately after applying the electric field, the droplets began to coalesce due to the induced dipole-dipole interactions, resulting in coarsening of the structure. When this blend was subjected to higher electric fields, not only the coalescence of droplets, but also the formation of columns took place [Fig. 3(b)]. The coalescence rate increased with both the amplitude of the ac electric field and the density of droplets. This is because the dipole-dipole interaction is proportional to the square of the applied electric field and is inversely proportional to the fourth power of the distance between two droplets, as will be described later.

In these experiments we have succeeded in observing the coalescence process of two droplets, as shown in Fig. 4. A pair of droplets elongated along the electric field approach each other to reduce the interdroplet distance and then coalesce into a large droplet. Immediately after coalescence the droplet is strongly deformed, but then gradually relaxes to an equilibrium shape.

Under electric fields droplets are elongated and become prolate spheroids. The lengths of the major and minor axes were estimated from the normalized spatial correlation function, defined as

$$
C(\boldsymbol{r})=\frac{\langle S(\boldsymbol{r}) S(0)\rangle-\langle S(0)\rangle^{2}}{\left\langle S(0)^{2}\right\rangle-\langle S(0)\rangle^{2}},
$$

where $S(\boldsymbol{r})=1$ in the bright region (LCP) and 0 in the dark region $(\mathrm{PIB})$, and $\langle\cdots\rangle$ means the spatial average. Clearly, $C(\boldsymbol{r})$ is anisotropic for spheroids. We can define two correlation lengths, $\xi_{c}$ and $\xi_{a}$, which correspond to the major and minor axes, respectively, as follows. In 3D space, $C(\boldsymbol{r})$ takes
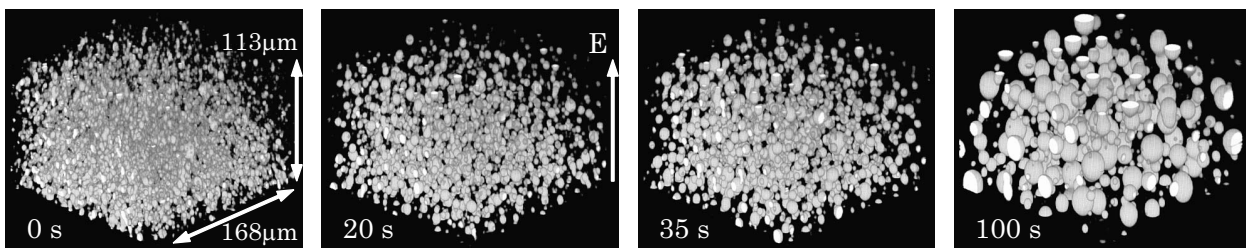

(a) $2 \mathrm{kV}_{\mathrm{amp}} / \mathrm{mm}$
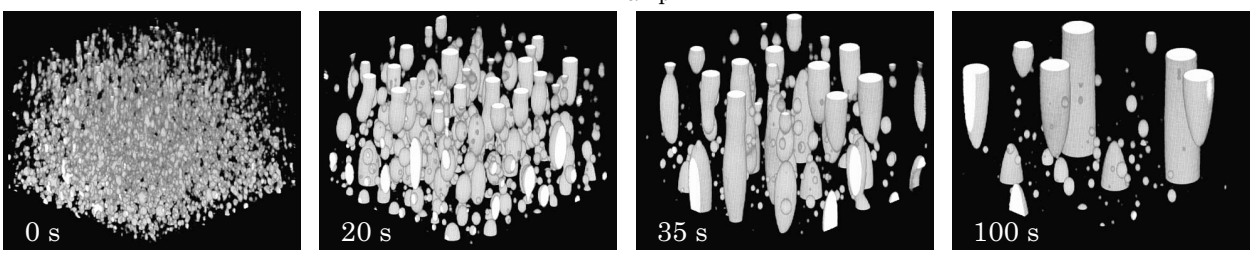

FIG. 3. Morphological changes after applying ac electric fields of (a) 2 and (b) $4 \mathrm{kV}_{\mathrm{amp}} / \mathrm{mm}$ with a frequency of $512 \mathrm{~Hz}$. The electric fields were applied at $t=0$. The deformation defined as $D=(c-a) /$ $(c+a)$ was calculated for each image. In (a) $D=0.0$ at 0,20 , and 35 s, $D=0.1$ at $100 \mathrm{~s}$, and in (b) $D$ $=0.0$ at $0 \mathrm{~s}, D=0.32$ at $20 \mathrm{~s}, D$ $=0.5$ at $35 \mathrm{~s}, D$ is impossible to calculate at $100 \mathrm{~s}$ because a columnar structure is formed.

(b) $4 \mathrm{kV}_{\mathrm{amp}} / \mathrm{mm}$ 

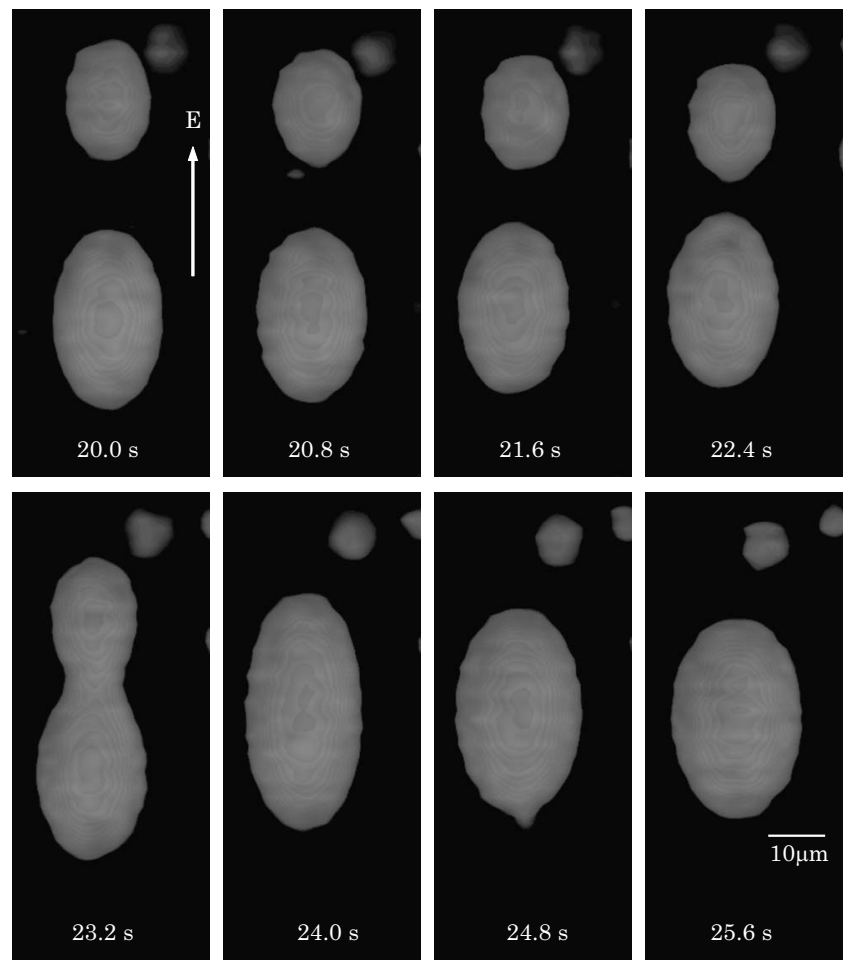

FIG. 4. Sequential images taken during the coalescence process of a pair of droplets in an electric field of $3 \mathrm{kV}_{\mathrm{amp}} / \mathrm{mm}$.

the maximum of 1 at the origin and monotonically decreases. The correlation lengths $\xi_{c}$ and $\xi_{a}$ are defined as the distances along the major and minor axes at which the correlation function equals $1 / 2$. These correlation lengths can be related to the lengths of the major and minor axes, $c$ and $a$, by $c$ $\cong 1.44 \xi_{c}$ and $a \cong 1.44 \xi_{a}$ (see the Appendix), where we have assumed that spheroids with the same aspect ratio and size are randomly and sparsely dispersed. In our results, however, the droplet shape and size have some degree of distribution, so the obtained values of $c$ and $a$ should be regarded as averages. Figures 5(a) and 5(b) show the time dependencies of $c$ and $a$, respectively, at several amplitudes of the ac electric field. In Fig. 5(b), we could not obtain the length of the major axis at extended times because of the formation of columns. In terms of the deformation, defined as $D=(c$ $-a) /(c+a)$, it was found that $D$ increased with time, and the column formation started at approximately $D=0.4$ almost irrespective of the applied electric field. Data for $D>0.4$ in Fig. 5(b) were not plotted, while in Fig. 5(a) all the data were plotted, and the data for $D \leq 0.4$ and $D>0.4$ are distinguished by open and closed symbols, respectively.

The growth rates of the lengths of both the major and minor axes increased when increasing the electric field. As can be seen in Fig. 5(a), the length of the minor axis, $a$, increases exponentially with time in the early stage $(D$ $\leq 0.4)$, but in the late stage $(D>0.4)$ the growth rate slows, and the size is almost constant. This indicates that once the columnar structure is formed there is hardly any coalescence of these columns. The initial slopes in Fig. 5(b) are steeper than those in Fig. 5(a) for the same electric field. This difference may be due to elongation. The droplet deformation $D$ when subjected to an electric field is proportional to the ra-
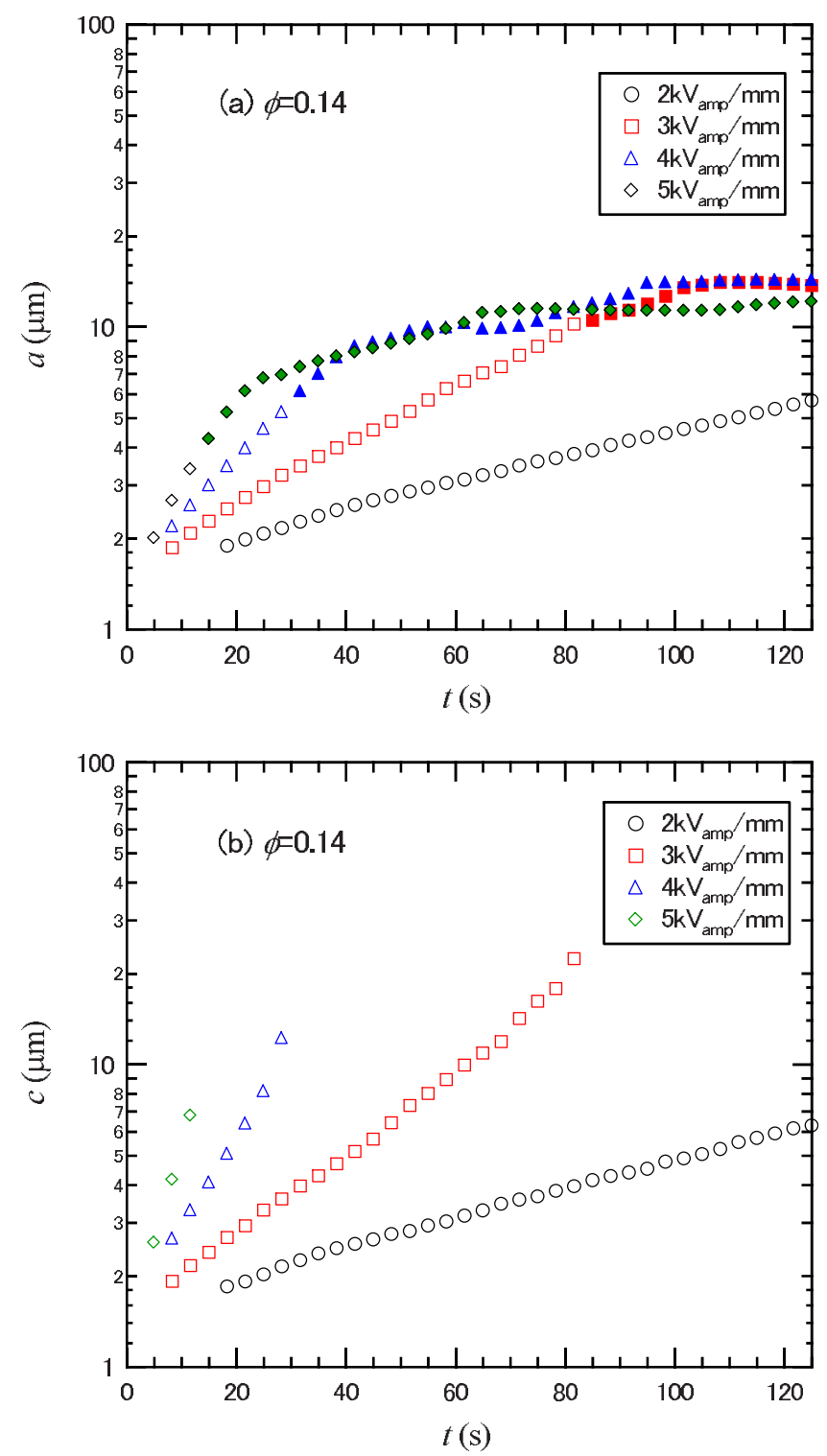

FIG. 5. (Color online) Time dependencies of (a) $a$ and (b) $c$ at several amplitudes of ac electric field for $\phi=0.14$ (LCP:PIB $=1: 6$ ). Open and closed symbols in (a) are for $D \leq 0.4$ and $D$ $>0.4$, respectively.

dius without the electric field [18]; hence, as droplets grow larger with time the growth rate of the major axis $c$ is accelerated while that of the minor axis $a$ is reduced. The exponential growth is discussed in detail in the next section.

\section{DISCUSSION}

First, let us consider a simple model without elongation, that is, all the droplets maintain a spherical shape during the coalescence process. From dimensional analysis the radius $r$ is given as

$$
r=r_{0} f\left(\frac{\varepsilon_{0} E_{\mathrm{rms}}^{2} t}{\eta}\right),
$$

where $r_{0}$ is the initial droplet radius, $\eta$ is the viscosity of $\mathrm{PIB}, \varepsilon_{0}$ is the dielectric constant of vacuum, $E_{\mathrm{rms}}$ is the root 

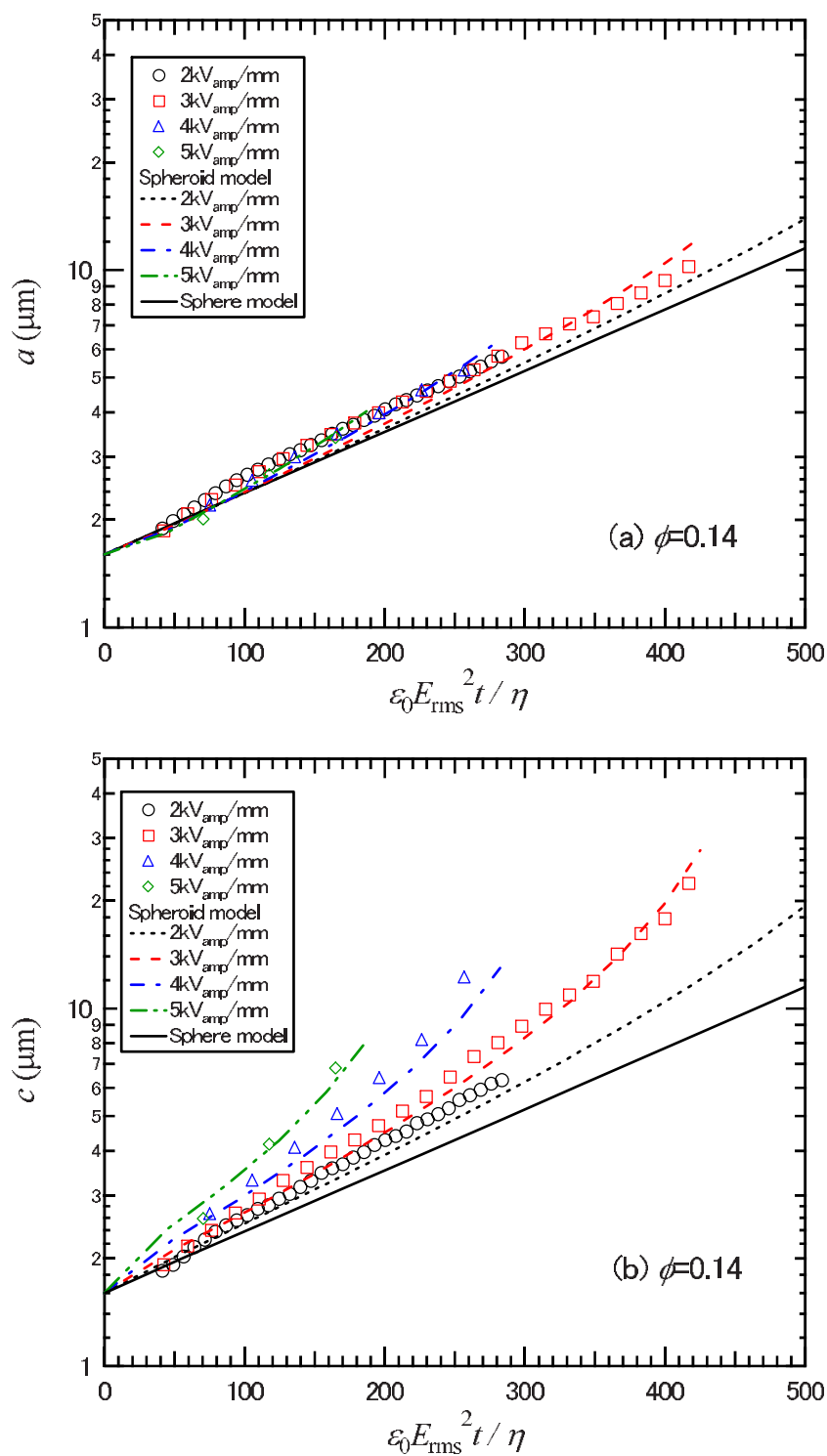

FIG. 6. (Color online) Plots of (a) $a$ and (b) $c$ vs $\varepsilon_{0} E_{\mathrm{rms}}^{2} t / \eta$ for $\phi=0.14$ (LCP:PIB=1:6). Theoretical values are also plotted for the spheroid and sphere models.

mean square of the ac electric field, and $f(x)$ is a scaling function. In Fig. 6 we replotted the data of Fig. 5 as a function of $\varepsilon_{0} E_{\mathrm{rms}}^{2} t / \eta$. Note that the initial radius $r_{0}$ at each value of electric field is almost the same in our experiments due to the applied preshear. The growth of $a$ seems to obey the scaling relation as shown in Fig. 6(a), while in Fig. 6(b) it appears that of $c$ does not. This is natural because the deformation $D$ increases with time, so that the structure at each time is not similar to the others, resulting in a breakdown of the scaling relation. Although the above simple model is not perfectly applicable to the present system, we can calculate the growth rate from the model to examine the volume fraction dependence and then compare these results to those of an improved model, which we propose, that takes into account this deformation.

In the calculation, we used a hierarchical model [15]. In this model, it is assumed that at $t=0$ spherical droplets of the same radius are uniformly dispersed, and that after time $T_{1}$ pairs of droplets coalesce to form larger spherical droplets with double the volume, and then these doublets coalesce to form droplets with quadruple the initial volume after a further time $T_{2}$, and so on. In order to determine the time $T_{n}$ required for droplets of radius $r_{n-1}=2^{(n-1) / 3} r_{0}$ to coalesce and form droplets of radius $r_{n}=2^{n / 3} r_{0}$, we consider the time evolution of the average distance between nearest-neighbor droplets, $R$.

The electrostatic interaction energy necessary to estimate the electrostatic attraction force between a pair of droplets is expressed by using the point-dipole approximation:

$$
u(r, \theta)=-\frac{1}{4 \pi \varepsilon_{0} \varepsilon_{2}} \frac{d^{2}}{R^{3}}\left(3 \cos ^{2} \theta-1\right),
$$

where $\theta$ is the angle between the electric field and a line between the two droplets, $\varepsilon_{2}$ is the relative dielectric constant of the matrix, and $d$ is the induced dipole moment:

$$
d=4 \pi \varepsilon_{0} \varepsilon_{2} \beta E r^{3},
$$

with

$$
\beta\left(\varepsilon_{1}, \varepsilon_{2}\right)=\frac{\varepsilon_{1}-\varepsilon_{2}}{\varepsilon_{1}+2 \varepsilon_{2}},
$$

where $\varepsilon_{1}$ is the relative dielectric constant of the droplets. In general, the effective polarizability $\beta\left(\varepsilon_{1}, \varepsilon_{2}\right)$ depends on the conductivities $\sigma_{1}$ (droplet) and $\sigma_{2}$ (matrix) and the frequency $f$ of the applied electric field; so $\varepsilon_{j}(j=1,2)$ in Eq. (3c) should be replaced with $\widetilde{\varepsilon}_{j}=\varepsilon_{j}+\sigma_{j} /\left(i 2 \pi \varepsilon_{0} f\right)$. To avoid conduction we used ac electric fields of $512 \mathrm{~Hz}$ in our experiments, at which $\left|\beta\left(\widetilde{\varepsilon}_{1}, \widetilde{\varepsilon}_{2}\right)\right| / \beta\left(\varepsilon_{1}, \varepsilon_{2}\right)=1.00004$.

For simplicity, putting $\theta=0$ in Eq. (3a), the electrostatic attraction force can be estimated as follows:

$$
F_{\text {electro }} \approx 24 \pi \frac{\varepsilon_{0} \varepsilon_{2} \beta^{2} E_{\mathrm{rms}}^{2} r^{6}}{R^{4}},
$$

where we have replaced $E$ in Eq. (3b) with $E_{\text {rms }}$ because the droplets cannot follow the oscillating electric field. Since the friction coefficient of a droplet of radius $r$ is $6 \pi \eta r$, the time evolution of $R$ is given by

$$
6 \pi \eta r \frac{d R}{d t}=-48 \pi \varepsilon_{0} \varepsilon_{2} \beta^{2} E_{\mathrm{rms}}^{2} r^{2}\left(\frac{r}{R}\right)^{4} .
$$

Now if we assume that after the $n$th step, that is, at time $t_{n}$ $=\sum_{i=1}^{n} T_{i}$, the droplets are homogeneously dispersed, then the average distance between droplets, $R_{n}$, is expressed as $R_{n}$ $=f \phi^{-1 / 3} r_{n}$, where $\phi$ and $r_{n}$ are the volume fraction of droplets and the radius of droplets after the $n$th step, respectively, and $f$ is a constant depending on the spatial distribution of the droplets. The solution of Eq. (5) is

$$
t-t_{n-1}=-\frac{\eta}{40 \varepsilon_{0} \varepsilon_{2} \beta^{2} E_{\mathrm{rms}}^{2} r_{n-1}^{5}}\left[R(t)^{5}-R_{n-1}^{5}\right],
$$

where we have used an initial condition of $R=R_{n-1}$ at $t$ $=t_{n-1}$. From this equation $T_{n}=t_{n}-t_{n-1}$ is easily obtained by putting $R\left(t_{n}\right)=0$, 


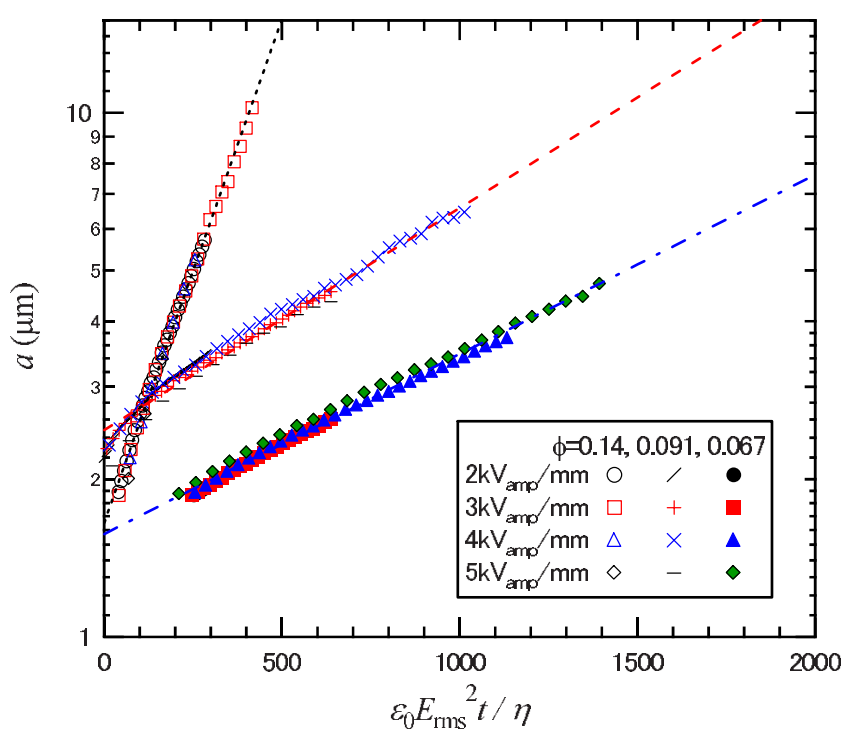

FIG. 7. (Color online) Plots of $a$ vs $\varepsilon_{0} E_{\mathrm{rms}}^{2} t / \eta$ under electric fields $E_{0}=2,3,4,5 \mathrm{kV}_{\mathrm{amp}} / \mathrm{mm}$ and at volume fractions $\phi=0.14$ (LCP:PIB=1:6), 0.091 (1:10), and 0.067 (1:14).

$$
T_{n}=\frac{\eta}{40 \varepsilon_{0} \varepsilon_{2} \beta^{2} E_{\mathrm{rms}}^{2}}\left(\frac{R_{n-1}}{r_{n-1}}\right)^{5}=C \frac{\eta}{\varepsilon_{0} \varepsilon_{2} \beta^{2} E_{\mathrm{rms}}^{2}} \phi^{-5 / 3},
$$

where $C=f^{5} / 40$. Note that $T_{n}$ is independent of the step for the sphere model, so that we define the characteristic time as $\tau=T_{n}$. Thus, $t\left(=t_{n}\right)=n \tau$ and $r\left(=r_{n}\right)=2^{n / 3} r_{0}$ yield

$$
r=2^{t / 3 \tau} r_{0}=r_{0} \exp \left(p \frac{\varepsilon_{0} E_{\mathrm{rms}}^{2} t}{\eta}\right),
$$

with

$$
p=\frac{\ln 2}{3 C} \varepsilon_{2} \beta^{2} \phi^{5 / 3},
$$

indicating that the radius increases exponentially with time, as seen in Fig. 6(a), and the growth rate depends on the volume fraction as $\phi^{5 / 3}$.

We measured the volume fraction dependence of $p$ to confirm whether Eq. (8b) holds, despite the aforementioned limitations of this simplified model. Figure 7 shows a semilogarithmic plot of the length of the minor axis $a$ versus the scaled time $\varepsilon_{0} E_{\mathrm{rms}}^{2} t / \eta$ under various electric fields $E_{0}$ $=2,3,4,5 \mathrm{kV}_{\mathrm{amp}} / \mathrm{mm}$ and for volume fractions $\phi=0.14$ (LCP:PIB = 1:6), $0.091(1: 10)$, and 0.067 (1:14). The data at each volume fraction fall almost on a straight line irrespective of the electric field, and the slope increases with the volume fraction, as predicted from the above theory. Figure 8 shows the volume fraction dependence of the slope $p$ obtained from Fig. 7, where the measurements were repeated twice at the same volume fraction. The slope $p$ seems to obey a power law with an exponent of $5 / 3$. By using the least-squares method the coefficient $C$ in Eq. (8b) was determined as $C \approx 2.2$, which is used for the following analyses.

Calculated values from Eq. (8) with $C \approx 2.2$ and $\phi=0.14$ are plotted as solid lines in Figs. 6(a) and 6(b), where the two lines are the same because we assumed that droplets are

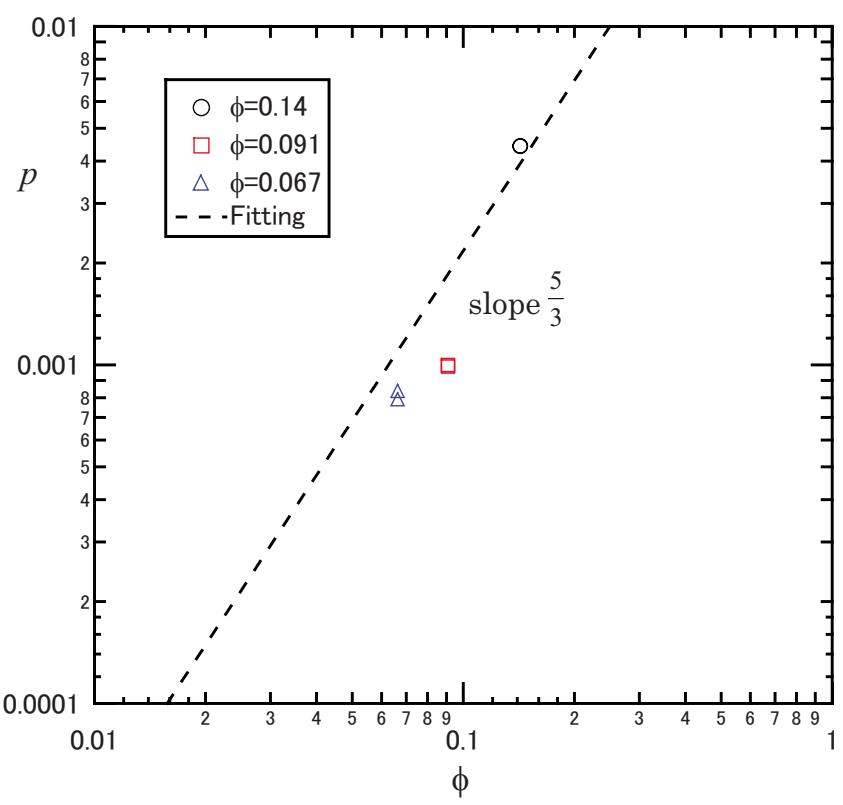

FIG. 8. (Color online) Dependence of the slope $p$ on the volume fraction $\phi$ in Eq. (8a).

spherical. The discrepancy between the experiment and the theory in Fig. 6(b) may arise from this assumption. In the discussion that follows, we will take into account the droplet deformation under electric fields.

According to Torza et al., the time-averaged deformation of a droplet under an ac electric field, $D=(c-a) /(c+a)$, is given theoretically as [18]

$$
D=\frac{9 \varepsilon_{0} \varepsilon_{2}}{16 \Gamma} \Phi E_{\mathrm{rms}}^{2} r
$$

where $\Phi$ is a function of dielectric constants, conductivities, viscosities, and the frequency of the ac electric field; $\Gamma$ is the interfacial tension; and $r$ is the radius of the droplet without an electric field. Note that the deformation $D$ oscillates in an ac electric field, but it is negligible in our experiments. Equation (9) means that the deformation is in proportion to the square of the electric field and the radius of droplet. From the experimental values of $c$ and $a$ we calculated $D=(c-a) /(c$ $+a)$ and $r=\sqrt[3]{a^{2} c}$ at various electric fields and volume fractions, and plotted $D$ vs $E_{\mathrm{rms}}^{2} r$ in Fig. 9. $D$ is almost proportional to $E_{\mathrm{rms}}^{2} r$, although the data are scattered to some degree. The proportionality constant was determined as $(5.3 \pm 0.1) \times 10^{-9} \mathrm{mV}_{\mathrm{rms}}^{-2}$ by the least-squares method. We will use this constant for the following numerical calculations to obtain the deformation at a given electric field.

For a spheroid the effective polarizability $\beta$ in Eq. (3c) should be modified [19]:

$$
\beta=\frac{1}{3} \frac{\varepsilon_{1}-\varepsilon_{2}}{\varepsilon_{1}+\left(\varepsilon_{1}-\varepsilon_{2}\right) n^{(z)}},
$$

with 


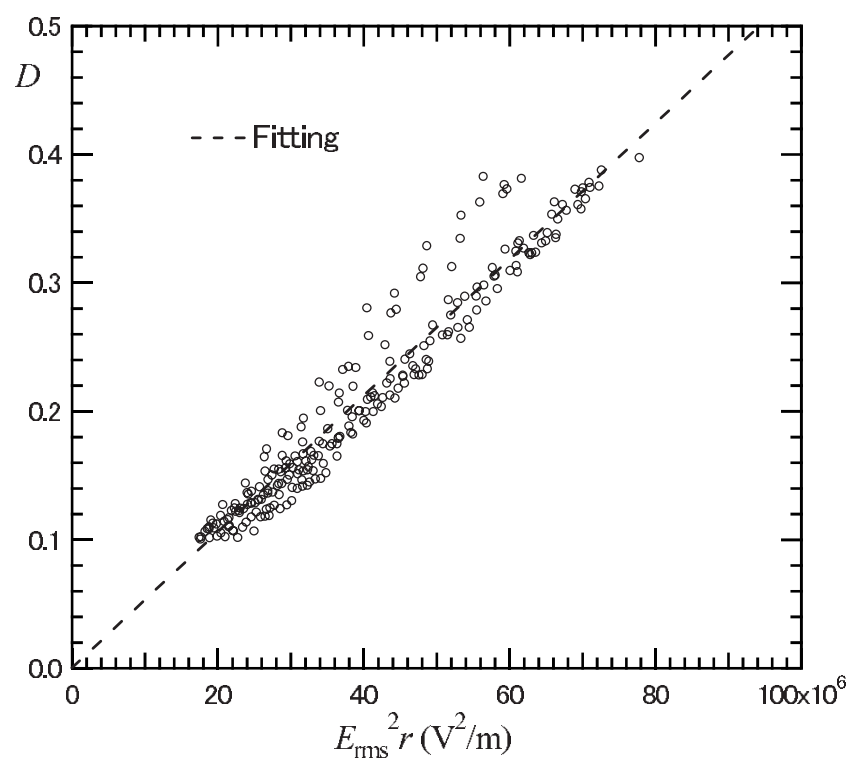

FIG. 9. Plot of $D$ vs $E_{\mathrm{rms}}^{2} r$ under electric fields $E_{0}$ $=3,4,5 \mathrm{kV}_{\mathrm{amp}} / \mathrm{mm}$ and at volume fractions $\phi=0.14,0.091,0.067$.

$$
n^{(z)}=\frac{1-e^{2}}{2 e^{3}}\left(\ln \frac{1+e}{1-e}-2 e\right),
$$

where $e$ is the eccentricity, defined as

$$
e=\sqrt{1-\frac{a^{2}}{c^{2}}}=\sqrt{1-\left(\frac{1-D}{1+D}\right)^{2}} .
$$

Since $\beta$ is a function of $D$, which increases with time, $T_{n}$ in Eq. (7) depends on the step. From the above equations, it is easily understood that $\beta$ is an increasing function of $D$, so that the growth rate should be accelerated with time, resulting in a deviation from the exponential growth for the simple sphere model. In the spheroid model we have to add $T_{i}$. At $t_{n}=\sum_{i=1}^{n} T_{i}$, the lengths of the major and minor axes are obtained from $D=(c-a) /(c+a), r=\sqrt[3]{a^{2} c}$, and $r\left(=r_{n}\right)=2^{n / 3} r_{0}$ as

$$
\begin{aligned}
& a=2^{n / 3} r_{0}\left(\frac{1-D}{1+D}\right)^{1 / 3}, \\
& c=2^{n / 3} r_{0}\left(\frac{1+D}{1-D}\right)^{2 / 3},
\end{aligned}
$$

where $D$ can be calculated using $(5.3 \pm 0.1) \times 10^{-9} \mathrm{mV}_{\mathrm{rms}}^{-2}$. In the calculation of $T_{i}$, for simplicity we use Eq. (7) for the sphere model, although the friction coefficient should be modified in Eq. (5).

Figures 10(a) and 10(b) show the time dependencies of $a$ and $c$, respectively, calculated at several amplitudes of the ac electric field for $\phi=0.14$. Dotted lines are added for the sphere model. As for the length of the minor axis $a$, the deviation from the sphere model is small, whereas the length of the major axis $c$ deviates from the sphere model significantly as the electric field is increased. It is now clear why $a$ almost obeys exponential growth but $c$ does not: the increase in the deformation $D$ accelerates the growth rate of droplet volume, while it reduces the growth rate of $a$, resulting in a
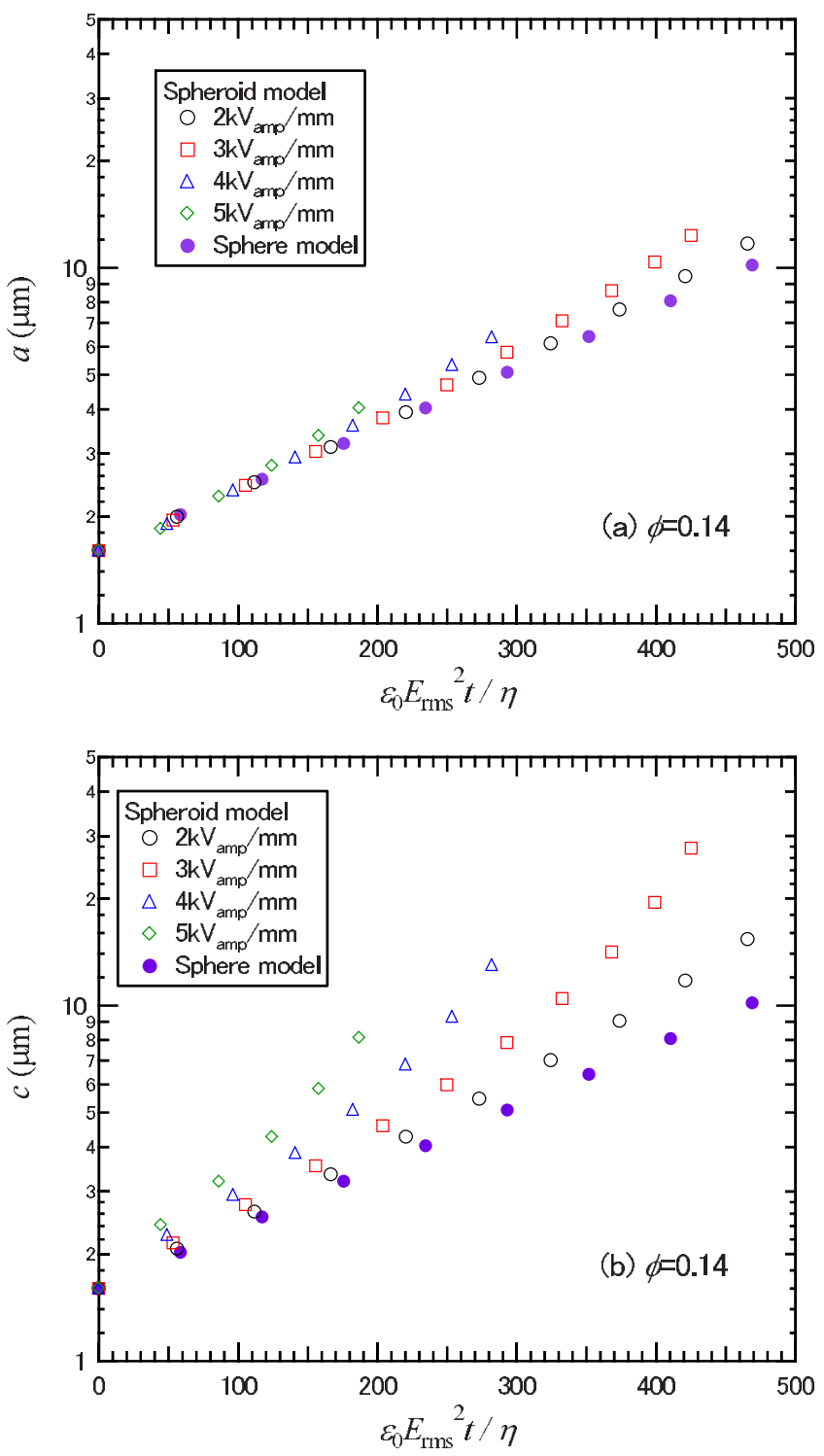

FIG. 10. (Color online) Time dependencies of (a) $a$ and (b) $c$ at several amplitudes of ac electric fields calculated from the spheroid model for $\phi=0.14$ (LCP:PIB=1:6). Results for the sphere model are also plotted.

cancellation. In contrast, the growth rate of $c$ is further accelerated. We plotted these theoretical values as broken lines in Fig. 6 to compare to the experimental results, and a good agreement is clearly seen, validating the spheroid model.

\section{CONCLUSIONS}

We have succeeded in observing the coalescence process in droplet-dispersed immiscible blends in three dimensions when subjected to a step electric field. Immediately after applying the electric field, the droplets began to coalesce due to the induced dipole-dipole interactions, and the average droplet size increased with time. In addition, the droplets were elongated along the electric field, and the deformation increased with the elapsed time. The length of the minor axis grew almost exponentially with time, while that of the major 
axis grew faster than exponential. For the model, we first derived the exponential growth from a simple sphere model where all the droplets were assumed to be spherical and droplet growth obeyed the hierarchical model. Results showed that the growth rate is proportional to the square of the applied electric field and the volume fraction to the power of 5/3, which was confirmed experimentally for the minor axis. Next, a spheroid model was proposed to incorporate the droplet deformation, which modifies the effective polarizability. The results from this model reproduced well the growth rates of both $c$ and $a$ obtained from the experiment.

\section{ACKNOWLEDGMENTS}

This work was partially supported by a Grant-in-Aid for Scientific Research [Priority Area "Soft Matter Physics" (Contract No. 463), C (Contracts No. 21540393 and No. 22540416), and Wakate B (Contract No. 21740306)] from the Ministry of Education, Culture, Sports, Science and Technology of Japan.

\section{APPENDIX: RELATION BETWEEN THE LENGTHS OF THE MAJOR AND MINOR AXES OF SPHEROIDS AND THE CORRELATION LENGTHS}

We can calculate the spatial correlation function of a spheroid-dispersed system by using the theory developed from the Avrami model [20] by Sekimoto [21]. This theory allows for the overlap of spheroids, but clearly in our system there is no overlap. In the theory, the spheroids (in the original theory the shape is arbitrary) are randomly distributed, so that some of them must overlap each other. The discrepancy between the model and our system may be resolved by only considering the case when the spheroids are sparsely dispersed. Therefore, we derive the correlation function at small volume fractions of spheroids.

We assume that the spheroids are of the same aspect ratio and size. According to the theory, the volume fraction of the spheroids, $\phi$, is given as

$$
\phi=1-\exp \left(-n V_{0}\right),
$$

where $n$ is the number density of spheroids and $V_{0}$ is the volume of a spheroid. Note that this equation is valid for all volume fractions. The spatial correlation function is expressed as

$$
\langle S(\boldsymbol{r}) S(0)\rangle=1-2(1-\phi)+(1-\phi)^{2} \exp \left[n V_{\text {over }}(\boldsymbol{r})\right],
$$

where $V_{\text {over }}(\boldsymbol{r})$ is the overlapped volume of two spheroids whose centers are at a distance $\boldsymbol{r}$ from each other. Therefore, the normalized correlation function defined in Eq. (1), $C(\boldsymbol{r})$, becomes

$$
C(\boldsymbol{r})=\frac{\exp \left[n V_{\text {over }}(\boldsymbol{r})\right]-1}{\exp \left(n V_{0}\right)-1}
$$

When the volume fraction is small, that is, $n V_{\text {over }}(\boldsymbol{r}) \leq n V_{0}$ $\ll 1$, this equation can be approximated as

$$
C(\boldsymbol{r})=\frac{V_{\mathrm{over}}(\boldsymbol{r})}{V_{0}} .
$$

This equation is independent of the number density, and therefore the two correlation lengths parallel and perpendicular to the applied electric field defined in the text can be related to the lengths of the major and minor axes of the spheroid: $c \cong 1.44 \xi_{c}$ and $a \cong 1.44 \xi_{a}$.
[1] K. Tajiri, K. Ohta, T. Nagaya, H. Orihara, Y. Ishibashi, M. Doi, and A. Inoue, J. Rheol. 41, 335 (1997).

[2] X. Pan and G. McKinley, J. Colloid Interface Sci. 195, 101 (1997).

[3] H. Kimura, K. Aikawa, Y. Masubuchi, J. Takimoto, K. Koyama, and K. Minagawa, Rheol. Acta 37, 54 (1998).

[4] J. Ha and S. Yang, J. Rheol. 44, 235 (2000).

[5] H. Orihara, T. Shibuya, T. Nagaya, and S. Ujiie, J. Phys. Soc. Jpn. 75, 063802 (2006).

[6] Y. H. Na, T. Shibuya, S. Ujiie, T. Nagaya, and H. Orihara, Phys. Rev. E 77, 041405 (2008).

[7] K. Aida, Y. H. Na, T. Nagaya, and H. Orihara, Phys. Rev. E 80, 041807 (2009).

[8] J. S. Eow, M. Ghadiri, A. O. Sharif, and T. J. Williams, Chem. Eng. J. 84, 173 (2001).

[9] J. S. Eow, M. Ghadiri, and A. Sharif, Colloids Surf., A 225, 193 (2003)

[10] M. Chiesa, J. A. Melheim, A. Pedersen, S. Ingebrigtsen, and C. Berg, Eur. J. Mech. B/Fluids 24, 717 (2005).
[11] D. J. Klingenberg, F. van Swol, and C. F. Zukoski, J. Chem Phys. 91, 7888 (1989).

[12] H. See and M. Doi, J. Phys. Soc. Jpn. 60, 2778 (1991).

[13] T. C. Halsey, J. E. Martin, and D. Adolf, Phys. Rev. Lett. 68, 1519 (1992).

[14] J. E. Martin, J. Odinek, and T. C. Halsey, Phys. Rev. Lett. 69, 1524 (1992).

[15] R. Botet, R. Jullien, and M. Kolb, J. Phys. A 17, L75 (1984)

[16] A. Inoue and S. Maniwa, J. Appl. Polym. Sci. 55, 113 (1995).

[17] See supplementary material at http://link.aps.org/supplemental/ 10.1103/PhysRevE.82.031805 for three-dimensional images after applying an ac electric field.

[18] S. Torza, R. G. Cox, and S. G. Mason, Philos. Trans. R. Soc. London, Ser. A 269, 295 (1971).

[19] L. D. Landau, E. M. Lifshitz, and L. P. Pitaevskii, Electrodynamics of Continuous Media, 2nd ed. (Pergamon, Oxford, 1984).

[20] M. Avrami, J. Chem. Phys. 9, 177 (1941).

[21] K. Sekimoto, Physica A 135, 328 (1986). 